\title{
COMBINATION OF THERMAL AND GEOMETRIC INFORMATION FOR BIM ENRICHMENT
}

\author{
Hélène Macher ${ }^{1}$ *, Marouane Boudhaim ${ }^{1}$, Pierre Grussenmeyer ${ }^{1}$, Monica Siroux $^{1}$, Tania Landes ${ }^{1}$ \\ ${ }^{1}$ ICube Laboratory UMR 7357, INSA Strasbourg, France \\ \{helene.macher, marouane.boudhaim, pierre.grussenmeyer, monica.siroux, tania.landes\}@insa-strasbourg.fr
}

Commission II, WG II/8

KEY WORDS: energy efficiency, infrared camera, RGB camera, photogrammetry, laser scanning, data fusion, BIM

\begin{abstract}
:
In the context of building renovation, infrared (IR) cameras are widely used to perform the energy audit of buildings. They allow analysing precisely the energetic performances of existing buildings and thermal analyses represent a key step for the reduction of energy consumption. They are also used to assess the thermal comfort of people living or working in a building. Building Information Models (BIM) are widespread to plan the rehabilitation of existing buildings and laser scanning is now commonly used to capture the geometry of buildings for as-built BIM creation. The combination of thermographic and geometric data presents a high number and variety of applications (Lagüela and Díaz-Vilariño, 2016). However, geometric and thermal information are generally acquired separately by different building stakeholders and thermal analyses are performed with independence of geometry. In this paper, the combination of thermal and geometric information is investigated for indoor of buildings. The aim of the project is to create 3D thermographic point clouds based on data acquired by a laser scanner and a thermal camera. Based on these point clouds, BIM models might be enriched with thermal information through the scan-to-BIM process.
\end{abstract}

\section{INTRODUCTION}

Building information models are widely used for building diagnostics and rehabilitation planning because of their ability to integrate all information provided by building stakeholders. The creation of an as-built BIM requires the acquisition of the geometry of the building. Laser scanning is now a common technique to achieve this goal. The so-called scan-to-BIM process which still remains largely manual is being automated (Macher et al., 2017).

In parallel, in the context of building renovation, it is also necessary to perform an energy audit of the building. Infrared thermography is a technique commonly used for this purpose. Infrared images have multiple uses. Indoor, they allow "perceiving » the temperature of different surfaces, which is relevant in multiple cases. They are used in data centers and farm servers, museums and old architectures which are sensitive to heat. Infrared cameras allow analyzing precisely the energetic performances of a building. As highlighted by Scaioni et al. (2017) the thermal analysis of buildings represents a key step for the reduction of energy consumption. Considering the plenty of existing buildings, a slight improvement of their energy efficiency may result in a large overall saving of energy.

Infrared cameras also allow assessing the thermal comfort of the people living or working in a building. Thermal comfort is defined as "that condition of mind which expresses satisfaction with the thermal environment" (ANSI/ASHRAE Standard 55, 2010). Thermal comfort or discomfort is a physiological feeling triggered by thermal sensors and receptors in the skin and the brain (Mayer, 1993) which give a signal when the temperature falls under a certain threshold or is above a certain threshold.
Traditionally, studying thermal comfort relies on the estimation of these thresholds, or the preferred body temperature. To do so, it is important to understand the factors that affect such body temperatures. These variables can be divided into mainly environmental and personal factors (Aucliciens and Szokolay, 2007). There are many models in literature that have been developed to assess it. One of these models, the adaptive model, has proven to be compatible with BIM methodologies (Boudhaim, 2018).

The inspection of a building requires normally the manual interpretation of the thermal images to detect heat loss (Gade and Moeslund, 2014). In the last few years, the thermal study of buildings is also under automation notably by combining thermal and metric information. Indeed, the geometry provides the exact location of defects and an accurate energy analysis. Since the geometric resolutions of infrared cameras and the number of strong features in thermal images are low, generating an accurate 3D model exclusively from thermal images is quite challenging (Hoegner et al., 2018). The information provided by a thermal camera is usually combined with data from another sensor. Data fusion can be considered either by creating multisensors systems (Hoegner et al., 2018; Vidas et al., 2013; Wang and Cho, 2015) or by combining data from different sensors in a later on (Lagüela et al., 2013; Lerma et al., 2011).

In this paper, a processing chain is proposed to create an accurate $3 \mathrm{D}$ thermal point cloud based on data acquired from a laser scanner and a thermal camera which has the ability to capture simultaneously IR and RGB images. The aim of our project is to automatically enrich BIM models with thermal information and to take advantage of such information for opening detection. The contribution focuses on indoor of

\footnotetext{
* Corresponding author
} 
buildings and more specifically on rooms. The overall acquisition of a building can be performed accurately and fast with a laser scanner. In order to assign thermal information to the point cloud obtained from the laser scanner, each room is acquired with a thermal camera. One should note that the segmentation of a point cloud into rooms was already proposed in Macher et al. (2017).

The paper will be composed of four main parts. The fundamental concept of measurements of an infrared camera will be first presented. The acquisition of a dataset with a thermal camera and a laser scanner will be then described. Next, the different calibrations of both infrared and digital cameras will be detailed. Finally, the developed processing chain will be explained. Based on RGB images, IR images and a point cloud issued from laser scanning, the proposed approach allows creating a mesh textured with IR information. This first results will be discussed and potential improvements will be proposed.

\section{INFRARED CAMERA: FUNDAMENTAL CONCEPT}

The use of infrared camera has become more and more widespread due to its numerous qualities: it is a real-time, harmless, contactless and non-invasive technology that outputs two-dimensional pictures that are easy to process. To this effect, the infrared camera measures the radiative heat flux. The heat flux is related to the temperature's surface, as stated by the law of Stefan-Boltzmann:

$$
W=\varepsilon \sigma T^{4}
$$

where $\mathrm{W}$ the heat flux density, $\varepsilon$ the emissivity, $\sigma$ StefanBoltzmann constant and $\mathrm{T}$ the surface temperature.

In theory, this radiative flux uses a wide spectral range, but its intensity peaks at a specific wavelength. This wavelength depends on the temperature, and is described by the law of Wien:

$$
\lambda_{\max }=\frac{0.029}{T}
$$

In practice, infrared cameras use mainly two spectral ranges, mid-wavelength infrared $(2 \mu \mathrm{m}$ to $5 \mu \mathrm{m})$ and long-wavelength infrared $(8 \mu \mathrm{m}$ to $14 \mu \mathrm{m})$. It is due to two reasons. In most cases, surfaces tend to radiate most in these two wavelengths. In the context of indoor thermal assessment, we are mainly interested in the long-wavelength infrared. The second reason is the low absorption of the air of the radiation within these spectral ranges. The thermal camera used in our project has, according to its manufacturer, a spectral range between $7.5 \mu \mathrm{m}$ and $13 \mu \mathrm{m}$.

\section{DATA ACQUISITION}

In order to study the combination of geometric and thermal data, acquisitions were performed with a thermal camera and a laser scanner. The acquisition details are presented in this section.

\subsection{Case study}

To start our study, a classroom of about 12 per 6 meters was considered as a case study. At the back of the room targets are fixed on the walls (Figure 1a). These targets, known in local coordinates, are useful to scale image-based point cloud and to align image-based and laser scanning point cloud. The point cloud of the classroom acquired by the laser scanner FARO Focus3D X330 is presented Figure 1b without ceiling for visual purpose.

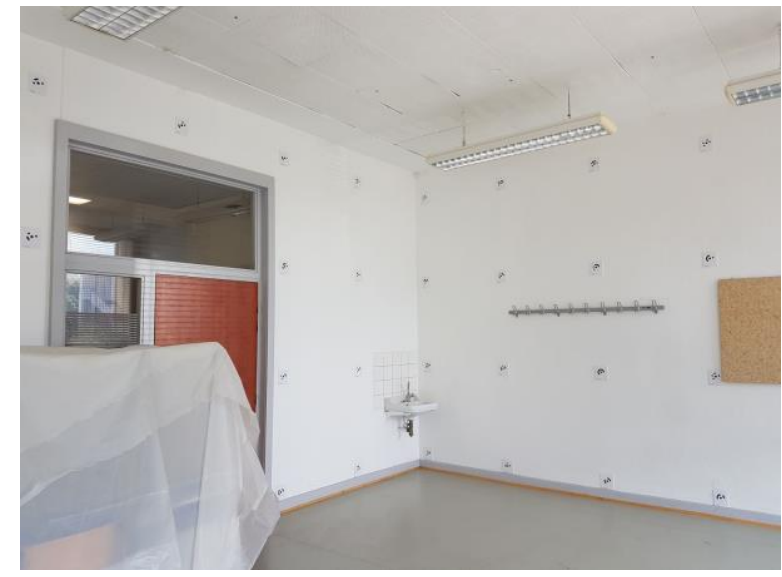

(a)

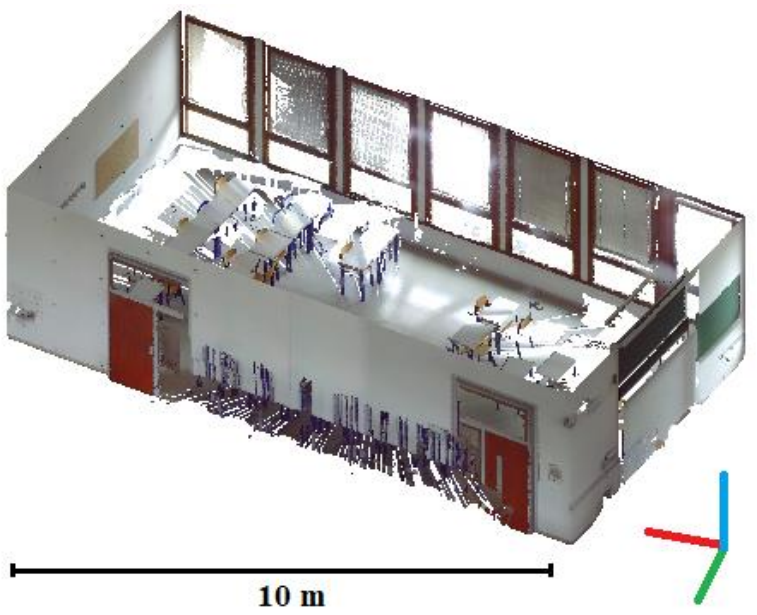

(b)

Figure 1. Classroom under study: (a) photograph of the back of the room with targets on walls; (b) laser scanning point cloud

\subsection{IR camera}

The thermal camera used for this study is the FLIR 450sc with an IR resolution of $320 \times 240$ pixels. For very accurate results, the manufacturer of the camera advises to wait 5 minutes after the start of the camera before measuring a temperature. Table 1 provides a few characteristics of this camera. It is composed of two sensors, a RGB camera with fixed focus and an infrared camera for which the focus can be set either automatically or manually.

\begin{tabular}{|c|c|}
\hline IR resolution & $320 \times 240$ pixels \\
Field of view (FOV) & $25^{\circ} \times 19^{\circ}$ \\
Focal length & $18 \mathrm{~mm}$ \\
Spectral range & $7.5-13 \mu \mathrm{m}$ \\
Accuracy & $\pm 2^{\circ} \mathrm{C}$ \\
Built-in digital camera & 3.1 Mpixel with LED light \\
Built-in digital lens data & FOV $53^{\circ} \times 41^{\circ}$ \\
\hline
\end{tabular}

Table 1. Characteristics of the thermal camera FLIR 450sc provided by the manufacturer 
The FLIR camera captures both thermal and RGB images at the same time. The visual images are used by the camera to enhance the clarity of thermal images by adding in visual details in real time. Additionally, an infrared image frame can be displayed on top of the visual image. In our study, the visual images will be used to deduce the thermal images' orientation as proposed by Alba et al. (2011). The camera is configured to save the thermal image and the visual image as separate JPEG files. Figure 2 presents an example of a thermal image and the corresponding photograph. The thermal camera resolution is lower therefore the thermal image only represents a part of the visual image. In the thermal image, the windows with awnings correspond to the maximum temperature whereas the wall parts between the windows produce the minimum temperature.

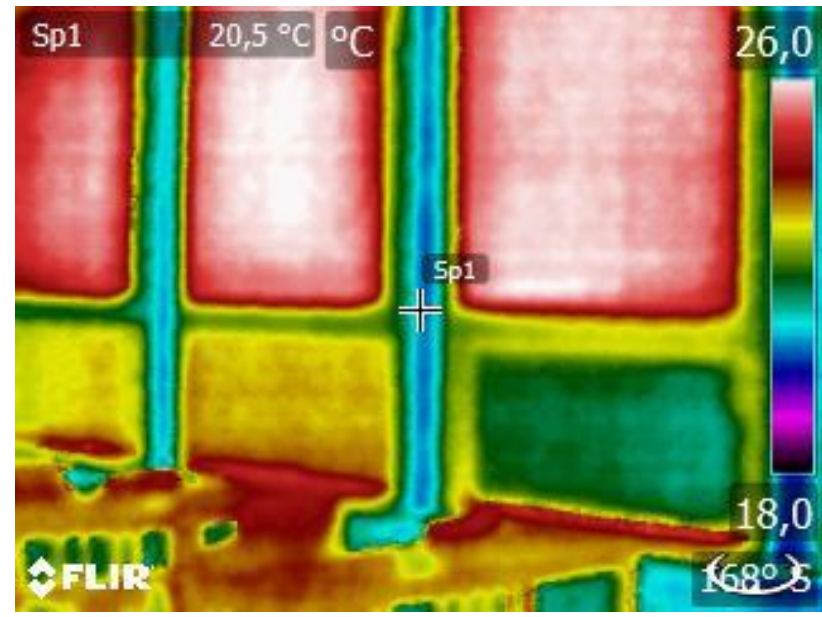

(a)

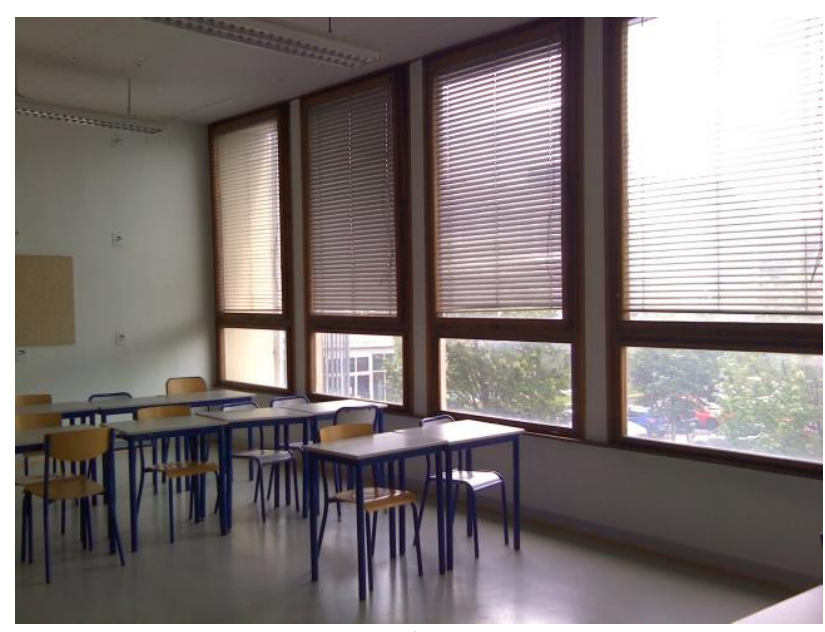

(b)

Figure 2. Data acquisition with the thermal camera: (a) infrared image (320×240); (b) corresponding RGB image $(2048 \times 1536)$

\subsection{Acquisition protocol}

Specific rules for indoor photogrammetry have to be followed for an accurate orientation of the images. One has to move around the room with the back against the wall and to stay as far as possible from the facing wall. Moreover, one should have enough overlapping between RGB images and ensure a small overlap of IR images not to have holes in the texture of the model. Three stripes were performed to cover the room with the IR information. Note that in our study, the ceiling and floor are not considered; the focus is made on thermal analysis of walls and openings.

Regarding IR images, the colour mapping of the pictures has to be fixed. Indeed, a thermal camera maps temperatures to colours, according to an «optimal » scale that is automatically calculated based on the visible temperature range. Therefore, the colours of a same surface may differ between two pictures, which will cause discontinuity for texturing. For the acquisition of the classroom, the scale was fixed from $18^{\circ} \mathrm{C}$ to $26^{\circ} \mathrm{C}$ which corresponds to the interval from the coldest temperature to the hottest temperature of the room.

The focus of the IR sensor can be set automatically (autofocus) by pushing a halfway down of the save button. In order to be able to perform a geometric calibration of the IR images, it was set manually. It would have been nice to set the focus on the hyperfocal distance. The necessity to consider depth of field very closely is an important constraint in close range photogrammetry (Kraus, 2011). However, with this camera, it is not possible to go back precisely to the previous focus if the user push by mistake a halfway down. For that reason the focus was set to infinity. For further acquisitions the possibility to connect the camera to an external device as for example a smartphone thanks to the Wi-Fi will be investigated. This will probably allow setting a fixed focus other than the infinity.

\section{CAMERA CALIBRATIONS}

Considering RGB and infrared images obtained with the thermal camera, several calibrations have to be performed: the geometric calibration of both sensors and the thermal calibration of the IR sensor.

\subsection{Geometric calibration of the RGB camera}

The geometric calibration of the RGB camera consists in taking a set of photographs around a checkerboard with white and black squares. The focus of the RGB sensor has to be the same as for data acquisition, which is the case since the focus is fixed. The multiple images of the checkerboard allow determining the camera parameters including camera intrinsics, extrinsics, and lens distortion parameters.

\subsection{Geometric calibration of the IR camera}

In order to perform the geometric calibration of the IR camera, a checkerboard was created specifically. With the checkerboard used for the calibration of the RGB camera there is not enough contrast between white and black squares on the IR image. To increase the contrast, the black squares were removed and replaced by aluminium paper as shown in Figure 3.

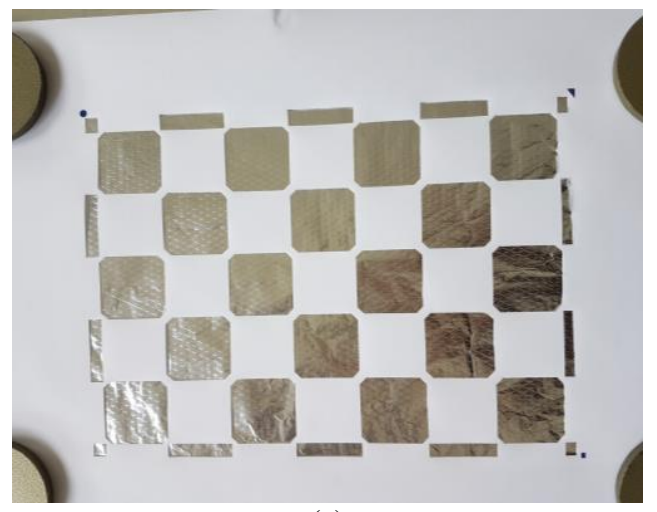

(a) 


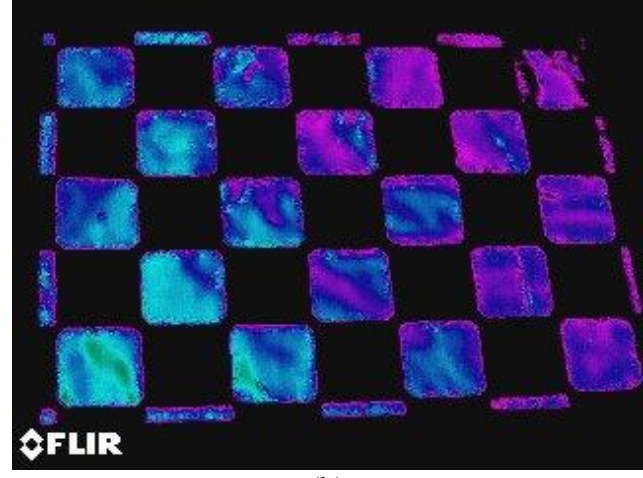

(b)

Figure 3. Checkerboard used for the geometric calibration of the IR camera: (a) photograph; (b) infrared image

The aluminium has the benefit to provide a high reflection of heat. During this calibration, a difference of about $1^{\circ} \mathrm{C}$ was observed on IR images between white and aluminium squares.

\subsection{Thermal calibration}

Beside the geometric calibration of the IR camera, one has to consider a thermal calibration of this sensor. For the calibration of the temperature measurements, there are two main parameters to take into account. The first and most important in effect is the material emissivity. While slight emissivity changes don't impact the temperature much, it is important in the case of low emissivity material as aluminum. The second parameter is reflected temperature, which is especially important for high reflectance material, which is in turn, characterized by low emissivity.

In our study, most indoor surfaces have an emissivity between 0.8 and 0.9 ; in this case the calibration has less impact. Moreover, in practical scenarios, rigorous calibration is often not performed, because of the lack of equipment or untrained users. Therefore, we chose in our study to not thermally calibrate the camera but, instead, to assess the error on the measured temperature. To this effect, we used a thermal probe, accurate to $0.1^{\circ} \mathrm{C}$, to verify the measurements.

We measured and compared the temperature in multiple points within the room. The infrared camera tends to underestimate the temperature by $0.0^{\circ} \mathrm{C}$ to $2.3^{\circ} \mathrm{C}$, with more than $80 \%$ of the surface that has less than $0.6^{\circ} \mathrm{C}$. After analysis of these errors, we didn't find any strong correlation between the surface material and the temperature discrepancy. Further analysis has led us to the reflected temperature factor. The surfaces that have shown the least discrepancy are the ones characterized by the most indirect lighting. It therefore causes these surfaces to underestimate less the real temperatures. The bias towards underestimating itself is attributed to the default emissivity used by the camera, 0.95 , which tends to be on average slightly higher than the indoor materials emissivity.

\section{CREATION OF A THERMAL POINT CLOUD}

Based on the RGB images, IR images and the point cloud issued from the laser scanner, a processing chain was developed so that thermal and geometric information can be combined. The processing chain is detailed in this section and the first result is shown and discussed.

\subsection{Processing chain}

Figure 4 presents an overview of the processing chain. The commercial software Metashape (Agisoft) was used. The exterior orientation of the RGB images is first calculated based on the matching of points of interest and orientation parameters are gathered. Due to the average quality of RGB images, some points were clicked manually to help the orientation. The scaling of the model is then performed thanks to the targets in the room. Once the exterior orientation and the scaling are carried out, a dense point cloud of the room is generated. Figure 5a presents the RGB images orientation and the dense point cloud of the classroom, which is produced from dense image matching.

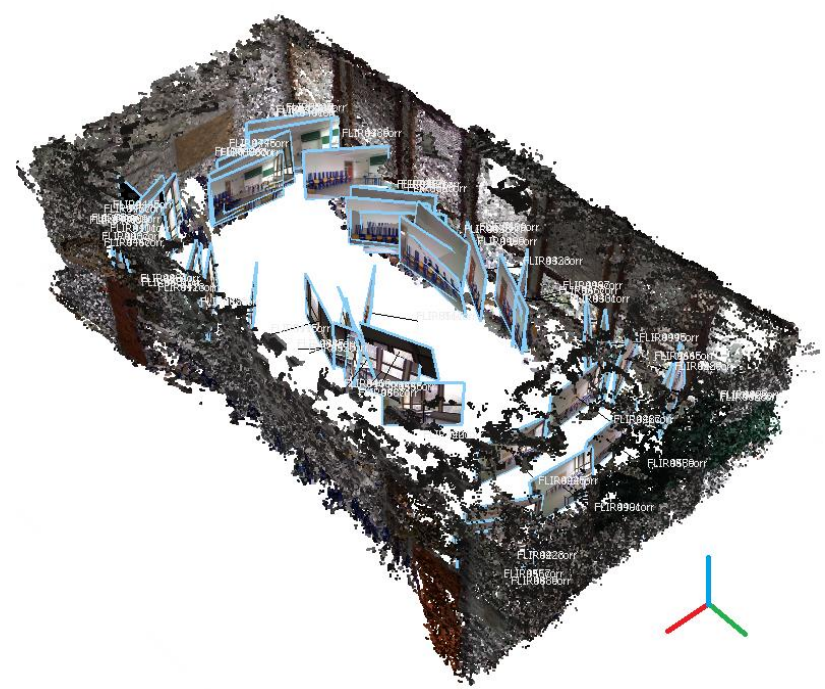

(a)

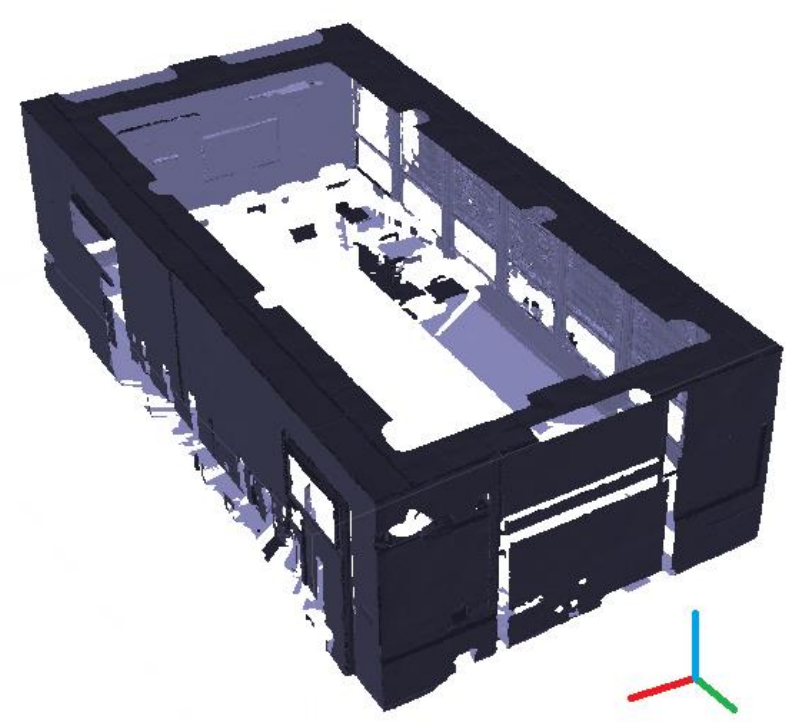

(b)

Figure 5. Data processing in Metashape software: (a) RGB images orientation and generated dense point cloud; (b) mesh created based on laser scanning point cloud 


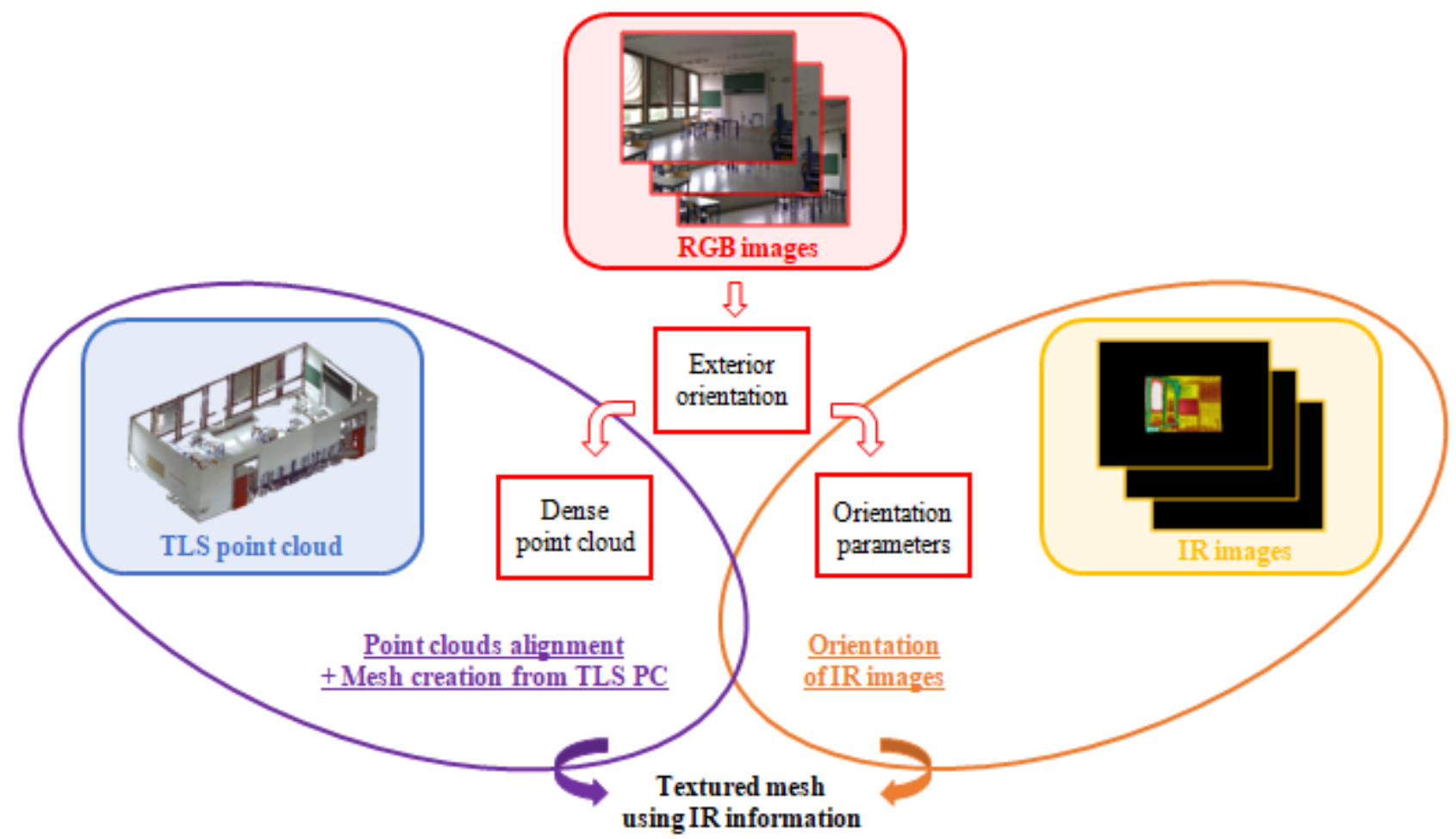

Figure 4. Overview of the processing chain

Each RGB image has a corresponding IR image. In order to apply the orientation parameters to the IR images, one had first to determine the position of the IR image in the RGB image. The centre of the IR image does not correspond to the one of the RGB image since the sensors are offset. A set of points were clicked within a IR and RGB image of a checkerboard to determine the affine transformation (translation, rotation and scale factor) to apply to the IR image to be positioned in the RGB image. Images of the size of RGB images were created with the IR images positioned on them. The orientation parameters determined before can be directly applied to these images.

The dense point cloud obtained with RGB images is not enough accurate to create a model based on it. The aim is to texture the laser scanning point cloud with the IR images. To do so, the image-based point cloud and the laser scanning point cloud were aligned manually in CloudCompare software (EDF R\&D). One should note that the scaling of the image-based point cloud combined with alignment of these two point clouds will be automated in future works. The image-based point cloud is used as a reference so that the laser scanning point cloud can be inserted in Metashape and textured by IR images.

Once the laser scanning point cloud is imported in Metashape software, the texturing is finally considered. The texturing of the point cloud was first tested but Metashape do not take into account the transparency of the image parts with no IR information and black colour is applied. Thus, a mesh of the laser scanning point cloud was created as illustrated Figure $5 \mathrm{~b}$. The point normals have to be oriented from the exterior to the interior of the room to perform properly the texturing. In this case, only IR information are applied during the texturing.
Note that the texturing of the point cloud will be reinvestigated in future work because it could be faster and it is more convenient to store the IR information in a point cloud considering the scan-to-BIM process.

\subsection{Results and discussion}

The mesh of the point cloud of the classroom under study issued from the laser scanner and textured with the infrared images is presented in Figure 6a. From a general point of view, the IR information is located more or less properly.

However, when looking at the wall with windows one can observe some problems in the texturing. Some images applied seem to be shifted or distorted. The photograph angle seems to have a huge influence on texturing quality. As illustrated in Figure $6 \mathrm{~b}$ and $6 \mathrm{c}$ the texturing is better for a photograph taken in front of windows than for a low angle photograph. Additionally, as shown by Teza and Pesci (2019), if the incidence angle is higher than $50^{\circ}$, the emissivity of materials is modified. Thus, for further acquisitions, one has to avoid taking very low angle photographs.

Beside the low-angle photographs, the problem of the texturing could come from the positioning of the IR image into the RGB image. An affine transformation has been considered but it will be better to apply a homographic transformation. 


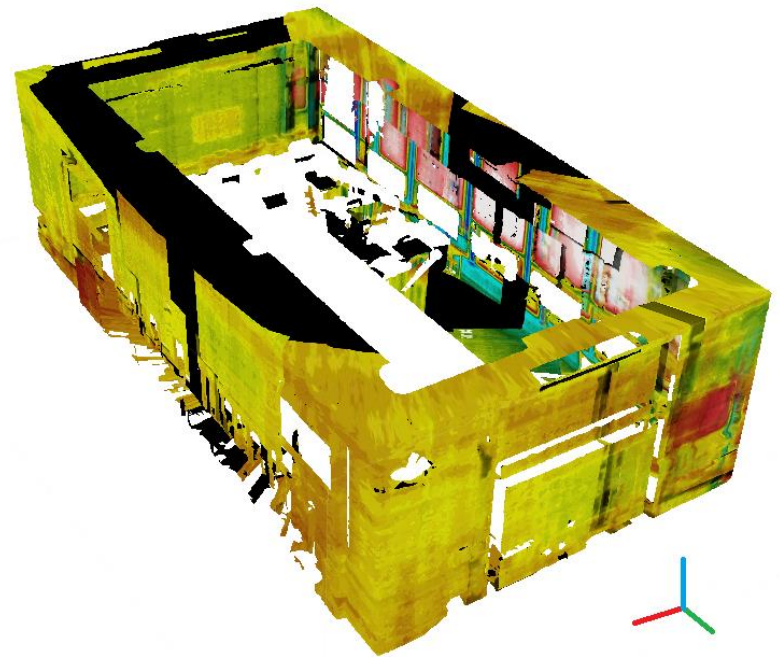

(a)

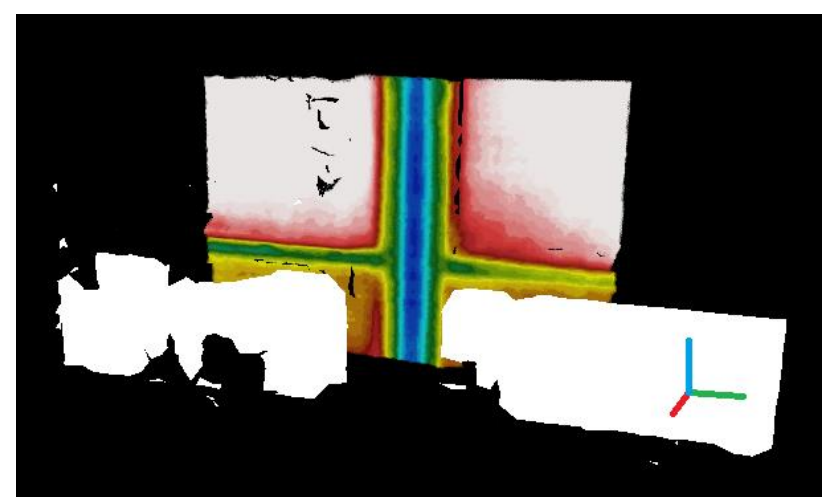

(b)

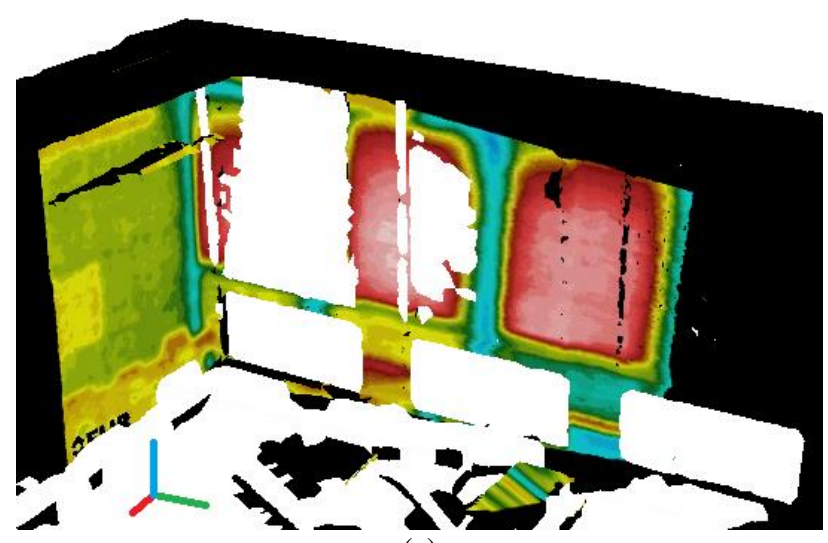

(c)

Figure 6. Textured mesh using IR images: (a) texturing of the whole mesh; (b) one image texturing - photograph in front of windows; (c) one image texturing - low angle photograph

\section{CONCLUSION AND FUTURE WORKS}

The investigation of a thermal camera, which has the ability to take thermal and RGB images simultaneously, was proposed for the combination of thermal and geometric information in indoor of buildings. First tests were conducted for a room.

The developed processing chain is based on RGB images, IR images and a point cloud issued from laser scanning and allows creating a mesh textured with IR information. Results are promising since the IR images taken with the thermal camera can be used to texture laser scanning data by exploiting RGB images.

However, a few improvements will be proposed in future work. Some steps of the processing chain are still partially manual and their automation will be studied. The compass information of the thermal camera will be exploited to improve the automation of RGB images orientation. Moreover, the alignment of the point cloud delivered by dense matching and the laser scanning point cloud can be automated by considering the height of the room and its contours.

Further investigations will be also conducted to solve the texturing problems. As explained in the previous section, one has to avoid low angle photographs and to determine properly the position of the IR image within the RGB image.

\section{ACKNOWLEDGEMENTS}

The authors would like to thank the master student Julian Rebetez involved in the acquisition and the processing of the data.

\section{REFERENCES}

Alba, M. I., Barazzetti, L., Scaioni, M., Rosina, E., and Previtali, M., 2011. Mapping infrared data on terrestrial laser scanning 3D models of buildings. Remote Sensing, 3(9), 18471870 .

Aucliciens, A., and Szokolay, S. V., 2007. Passive and Low Energy Architecture, International Design Tools and Techniques, University Of Queensland, Department of Architecture.

Boudhaim, M., 2018. Optical and thermal performance of complex fenestration systems in the context of building information modelling. Phd thesis.

Gade, R. and Moeslund, T. B., 2014. Thermal cameras and applications: a survey. Machine vision and applications, 25(1), 245-262.

Hoegner, L., Abmayr, T., Tosic, D., Turzer, S., and Stilla, U., 2018. Fusion of 3D point clouds with TIR images for indoor scene reconstruction. In: International Archives of the Photogrammetry, Remote Sensing and Spatial Information Sciences, ISPRS TC I Mid-term Symposium "Innovative Sensing - From Sensors to Methods and Applications", 10-12 October 2018, Karlsruhe, Germany, Volume XLII-1, pp. 189194.

Kraus, K., 2011. Photogrammetry: geometry from images and laser scans. Walter de Gruyter, p.60.

Lagüela, S. and Díaz-Vilariño, 2016. Thermographic 3D Modeling of Existing Constructions. In Non-destructive Techniques for the Evaluation of Structures and Infrastructure. CRC Press, Riveiro, B., and Solla, M. (Eds.), Chapter 12.

Lagüela, S., Díaz-Vilariño, L., Martínez, J., and Armesto, J., 2013. Automatic thermographic and RGB texture of as-built 
BIM for energy rehabilitation purposes. Automation in Construction, 31, 230-240.

Lerma, J. L., Cabrelles, M., and Portalés, C., 2011. Multitemporal thermal analysis to detect moisture on a building façade. Construction and Building Materials, 25(5), 21902197.

Macher, H., Landes, T., and Grussenmeyer, P., 2017. From Point Clouds to Building Information Models: 3D SemiAutomatic Reconstruction of Indoors of Existing Buildings. Applied Sciences, 7(10), 1030.

Mayer, E., 1993. Objective criteria for thermal comfort. Building and environment, 28(4), 399-403.

Scaioni, M., Rosina, E., L'Erario, A., and Dìaz-Vilariño, L., 2017. Integration of infrared thermography and photogrammetric surveying of built landscape. In: International Archives of the Photogrammetry, Remote Sensing and Spatial Information Sciences, GEOMATICS \& RESTORATION Conservation of Cultural Heritage in the Digital Era, 22-24 May 2017, Florence, Italy, Volume XLII-5/W1, pp. 153-160.

Teza, G., and Pesci, A., 2019. Evaluation of the temperature pattern of a complex body from thermal imaging and $3 \mathrm{D}$ information: A method and its MATLAB implementation. Infrared Physics \& Technology, 96, 228-237.

Vidas, S., Moghadam, P., and Bosse, M., 2013. 3D thermal mapping of building interiors using an RGB-D and thermal camera. In: 2013 IEEE International Conference on Robotics and Automation, Karlsruhe, Germany, May 6-10, 2013, pp. 2311-2318.

Wang, C., and Cho, Y. K., 2015. Performance evaluation of automatically generated BIM from laser scanner data for sustainability analyses. Procedia engineering, 118, 918-925. 